\title{
Caracterización y medición del nivel de gestión del conocimiento en las medianas y grandes empresas del Valle de Sugamuxi del Departamento de Boyacá
}

\author{
JOSÉ JAVIER GONZÁLEZ MILLÁN, M.A..,2,3 \\ Profesor Asistente, Universidad Pedagógica y Tecnológica de Colombia, Colombia. \\ javier.gonzalezmillan@uptc.edu.co \\ MIRYAM TERESA RODRÍGUEZ DÍAZ \\ Docente, Universidad Pedagógica y Tecnológica de Colombia, Colombia. \\ miryamteresa.rodriguez@uptc.edu.co \\ EDITH VIVIANA CÁRDENAS BARRERA \\ Administradora de Empresas, Universidad Pedagógica y Tecnológica de Colombia, Colombia. \\ edithviviana.cardenas@uptc.edu.co
}

\section{RESUMEN}

Este artículo presenta los resultados de una investigación de campo que buscaba determinar la situación actual de la gestión del conocimiento en medianas y grandes empresas del Valle de Sugamuxi en el Departamento de Boyacá (Colombia). Para ello se realizó un diagnóstico de sus procesos, se hizo una identificación cualitativa de los factores cuantitativos más relevantes y se propuso un modelo teórico para la gestión del conocimiento. El tipo de estudio fue el análisis descriptivo y ANOVA, regresión lineal y análisis de factores, encontrando que las empresas se encuentran en un grado medio de gestión del conocimiento.

Palabras clave. Gestión del conocimiento; empresa; indicadores; ANOvA; regresión.

Recibido: 30-jun-11, corregido: 25-abr-12 y aceptado: 26-jun-12

Clasificación JEL: M14

@

\footnotetext{
1 Este documento fue seleccionado en la convocatoria para enviar artículos, Call for Papers, realizada en el marco del "II Simposio Iberoamericano de Estudios Gerenciales: Una mirada interdisciplinar a la innovación", organizado por la revista académica Estudios Gerenciales bajo la dirección de la Facultad de Ciencias Administrativas y Económicas de la Universidad Icesi; el evento tuvo lugar los días 12, 13 y 14 de octubre de 2011, en la ciudad de Cali (Colombia). Este documento fue presentado en las sesiones simultáneas del área de "Innovación en gestión del conocimiento".

2 Este artículo es producto de la investigación intitulada "Medición del nivel de desarrollo e indicadores de la Gestión del Conocimiento, en las medianas y grandes empresas del Valle de Sugamuxi, Departamento de Boyacá" de la Universidad Pedagógica y Tecnológica de Colombia perteneciente a la línea de investigación de Gestión del conocimiento, del grupo de investigación Management.

3 Autor para correspondencia. Dirigir a: Calle 17 No 11ª̂16, Barrio San Martin, Sogamoso, Boyacá, Colombia.
} 


\section{Characterization and measurement of the level of knowledge management in medium-} sized and large companies in the Sugamuxi Valley in the State of Boyacá

This article presents the results of a field research project that aimed at determining the current situation of knowledge management in medium-sized and large companies in the Sugamuxi Valley in the State of Boyacá (Colombia). The project involved conducting a diagnosis of processes, making a qualitative identification of the most relevant quantitative factors, and proposing a theoretical model of knowledge management. This was an analytical descriptive study that used ANOva, linear regression, and factor analysis techniques for determining important variables, and demonstrated that companies are now at an average level when it comes to knowledge management.

Keywords. Knowledge management; business; indicators; ANOvA; regression.

\section{es}

\section{Caracterização e medição do nível de gestão do conhecimento em empresas de médio} e grande porte no Vale de Sugamuxi do Departamento de Boyacá

Isso apresenta os resultados de um estudo de campo que procurou determinar o estado atual da gestão do conhecimento em médias e grandes empresas do Vale de Sugamuxi no Departamento de Boyacá (Colômbia), ao mesmo tempo que foi feito um diagnóstico de processos, foi realizada uma identificação qualitativa dos fatores quantitativos mais relevantes e se propôs um modelo teórico para a gestão do conhecimento. O tipo de estudo foi análise descritiva e ANOvA, regressão linear e análise de fatores, a fim de encontrar variáveis importantes, descobrindo que as empresas estão em um nível médio de gestão do conhecimento.

Palavras-chave. Gestão do conhecimento; empresa; indicadores; ANOvA; regressão. 


\section{Introducción}

Hoy día, las organizaciones se han venido preocupando principalmente por retener el talento inmerso en sus empresas. De acuerdo con Clavijo (2011), la gestión del conocimiento cobra relevancia en las organizaciones modernas, hecho que permite la realización de un trabajo investigativo riguroso intitulado: "Medición del nivel de desarrollo e indicadores de la Gestión del Conocimiento, en las medianas y grandes empresas del Valle de Sugamuxi, Departamento de Boyacá". Los fundamentos epistemológicos de la investigación toman como referente las principales postulaciones de Nonaka \& Takeuchi (1995), bajo el modelo de Ciclo de Creación del Conocimiento.

La metodología utilizada pertenece al paradigma funcionalista plantado por $\mathrm{Du}$ rango (2005), el tipo y método de estudio es descriptivo e inductivo y la técnica estadística utilizada fue el censo, en donde se clasificaron cuarenta empresas de acuerdo con sus activos. Finalmente, como instrumento de recolección de información se contó con la encuesta estructurada, realizada a los gerentes de dichas compañías. El paquete estadístico SPSS ${ }^{\mathrm{mw}}$ (v.18) y ANOVA permitieron realizar un análisis descriptivo (frecuencias y tablas de contingencia), de regresión lineal y un análisis factorial (Díaz, 2002), respectivamente. La investigación da cuenta del nivel de desarrollo, respecto a la situación, los procesos de conversión y los factores relevantes de la gestión del conocimiento de orden cuantitativo es bajo con seis generaciones nuevas de productos y/o servicios; luego se puede afirmar que en materia de obtención de resultados, el desarrollo de la gestión del conocimiento es bajo.

Con respecto a los acápites más relevantes de la investigación, se encuentra, en primer lugar, realizar una revisión bibliográfica de las distintas teorías y procesos relacionados con la gestión del conocimiento; y posteriormente, hacer un análisis y caracterización tanto de la situación actual como de los procesos de la gestión del conocimiento empresarial. En segunda instancia, se determina la situación actual de la gestión del conocimiento en las medianas y grandes empresas del Valle de Sugamuxi. Un tercer momento lo constituye el diagnóstico a los factores organizacionales de índole cualitativo y cuantitativo más relevantes que inciden en la gestión del conocimiento. En cuarto lugar se presenta un análisis factorial por extracción de componentes principales donde se identifican variables relacionadas con la generación de nuevos productos y servicios y como tarea final, se concluye con el planteamiento de un modelo teórico de gestión del conocimiento aplicado a un sector empresarial concreto.

\section{La gestión del conocimiento}

Drucker (1993) hace ver el valor del conocimiento como un aspecto significativo para las organizaciones, es decir, como uno de los recursos relevantes en una compañía; por tanto, hoy se aúnan esfuerzos por definir cómo adquirirlo, retenerlo y administrarlo. Dicho planteamiento coincide con autores como García \& Cordero (2008) y Fernández \& Cordero (2010) quienes presentan como prioridad los procesos que conlleven a gestionar el conocimiento a todos los niveles corporativos como una cultura inmersa en la institución.

De otra parte, González (2009) y García, Domínguez \& Sánchez (2005) conciben la gestión del conocimiento como un ciclo, debido a su alta velocidad de cambio. Por su parte, David \& Foray (2002) consideran el conocimiento en términos económicos y contables; Medellín (2008) considera que la gestión del conocimiento debe constituirse 
como un baluarte de la alineación y direccionamiento estratégico, mientras que Swan \& Scarbrough (2001) y Díaz (2003) centran su atención en algunas fases neurálgicas del proceso, como la codificación y captura, a través del uso de las herramientas informáticas.

Finalmente, Sveiby (1997) describe la gestión del conocimiento mediante dos vertientes relacionadas con la línea de influencia, las cuales, originalmente, van desde una visión ingenieril a una humanística: la gestión del personal y la gestión de la información. Sin embargo, al profundizar un poco más, aparece una tercera corriente centrada en la organización. A continuación se hace una revisión de cada uno de estos postulados.

\subsection{Perspectiva de la información}

Moreno (2000) y Plaz (2003) presentan esta perspectiva como aquella que recoge la visión de Bill Gates, en la cual se presenta un ciclo de administración y tratamiento de la información dentro de la organización mediante mecanismos de asimilación y captación para presentar soluciones prácticas. También refuerzan esta percepción, Bernal (2005) y Pavez (2001), al considerarla como "el proceso organizacional que busca la combinación sinérgica del tratamiento de datos e información, a través de las capacidades de las tecnologías de información y de creatividad e innovación de los seres humanos" (Pavez, 2001, p. 21); evidenciando así un proceso sistemático para organizar, filtrar y presentar la información con el objetivo de mejorar la comprensión de las personas en un área específica de interés. Finalmente, Davenport (1997), con base en dichas apreciaciones, afirma que la información, relacionada con la tecnología de información, está estrechamente vinculada con la gestión del conocimiento.

\subsection{Perspectiva de proceso}

Zorrilla (1997), al citar a Quintas, la define como "el proceso de administrar continuamente conocimiento de todo tipo para satisfacer las necesidades presentes y futuras, para identificar y explotar recursos de conocimiento con el fin de alcanzar los objetivos organizacionales" (p. 2). Por su parte, Clemmons (2002), al citar a Machintosh, la conceptúa como "un proceso sistemático de la organización para alcanzar el éxito mediante la creación, la captación y el compartimento del conocimiento" (p. 9); de la misma manera, García (2002) la presenta como "un proceso de gestionar explícitamente los activos no materiales y existe para que la empresa pueda generar, buscar, almacenar y transferir el conocimiento y así conseguir aumentar la productividad y la competitividad" (p. 2). Al respecto, Shanhong (2002) y Rodríguez, Araujo \& Urrutia (2001) sustentan esta perspectiva basada en el ciclo de planear, organizar, coordinar y controlar las diversas actividades que conllevan a la creación y difusión de conocimiento, de manera eficiente, en una empresa o cualquier otro tipo de organización. De lo anterior se puede concluir que la gestión del conocimiento obedece a un ciclo lógico que, en términos generales, comienza con la identificación, creación, captura, compartición, almacenamiento y transferencia de conocimiento, ya sea tácito o explícito.

\subsection{Perspectiva humanística}

Se fundamenta en el capital intelectual mediante el direccionamiento del esfuerzo humano de la organización, propiciando el avance entre fase y fase del ciclo de conocimiento. Sveiby (1998), Serradell \& Pérez (2000) afirman que "la gestión del capital intelectual en una organización es importante, dado que la finalidad es añadir valor a los productos y servicios que ofrece la 
organización en el mercado y diferenciarlos competitivamente" (Serradell \& Perez, 2000, p.5). Garrido (2002), Grau (2001) y SaintOnge (Pavez, 2001) refuerzan este concepto basados en la habilidad que se tiene para poder desarrollar, mantener, influtenciar y renovar los activos intangibles, también denominados capital intelectual. Adicionalmente, Arbonies (2006) considera la gestión del conocimiento como un conjunto de disciplinas de la administración que trata el capital intelectual como un activo de la empresa. Lo anterior permite a los autores definir la gestión del conocimiento como el proceso de creación, captura, distribución, compartición, asimilación, explotación, uso y renovación del conocimiento como elemento generador y valor agregado en las organizaciones para hacerlas más competitivas utilizando el capital humano.

\subsection{Ciclo del conocimiento}

De acuerdo con el objeto de estudio propuesto y basados en las teorías de Nonaka \& Takeuchi (ver Gráfico 1) y Polanyi (1962), se tomó como referente el ciclo de conversión de conocimiento en el cual existen dos clases de conocimiento: el tácito y el explícito. El primero es aquel que está en la memoria del individuo en el transcurso de las experiencias para darlo a conocer en un determinado grupo de personas; consta de acciones y comportamientos que no se pueden explicar, reconocer o transmitir; en tanto que el segundo, se refiere al conocimiento que ha sido almacenado en algún tipo de medio como memorias, textos, periódicos o en sistemas de información y que puede ser transmitido en cualquier momento.

Por otro lado, el modelo planteado por Valhondon (2003) se fundamenta en un esquema de socialización (compartir experiencia) de externalización (formular conceptos), continua con la combinación (conocimiento sistémico) y por último, aparece la fase de interiorización (traducción a comportamiento diario y operaciones). Es pertinente aclarar que algunos autores, como Fresno (2001), presentan una quinta fase denominada asimilación, referida bá-

Gráfico 1. Ciclo de creación del conocimiento

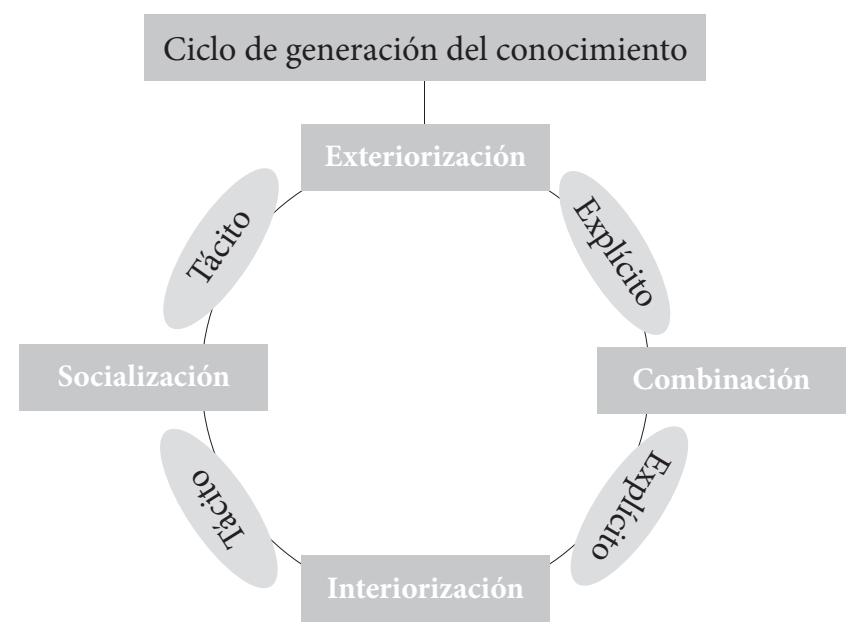

Fuente: Adaptado de Nonaka \& Takeuchi (1995, p. 62). 
sicamente, a la síntesis de experiencias en las bases cognitivas, ya sea del grupo o del trabajador.

\section{Metodología}

Se utilizó el estudio descriptivo mediante un proceso de diagnóstico de los elementos y la correlación de variables relevantes en la situación actual de la gestión del conocimiento. La metodología empleada fue la inductiva, ya que el análisis de los datos obtenidos permite acceder al planteamiento de indicadores con el fin de medir el nivel de desarrollo. Este método de análisis permite apreciar el comportamiento de las variables más importantes de la gestión del conocimiento en las empresas identificando las consecuencias, generadas por ellas, así mismo se utilizo el análisis factorial por el método de factores comunes (Díaz, 2002).

Se consideró como fuente primaria a los gerentes de las medianas y grandes empresas del Valle de Sugamuxi, para lo cual se hizo una encuesta guiada, ya que la terminología empleada no es muy conocida en el medio. En lo referente al instrumento de recolección de la información, se utilizó la encuesta de auto-diagnóstico ${ }^{4}$ adaptada de Carrillo (2006). En las fuentes secundarias se tomó como referente los documentos escritos y digitales, páginas de internet, informes estadísticos e investigaciones. La técnica utilizada fue la consulta de bases de datos, revistas, documentos e informes.

Se llevó a cabo un censo con las cuarenta medianas y grandes empresas de todos los sectores económicos del Valle de Sugamuxi teniendo como referencia el valor de los activos ya que no fue posible acceder al número de empleados. Con el fin de clasificar las empresas, se tuvo en cuenta la Ley

4 El cuestionario utilizado para este estudio puede ser asequible al lector interesado bajo solicitud directa a los autores.
1111 (Congreso de la Republica, 2006), que estipula un margen Unidades de Valor Tributario (UVT), para las que sean consideran empresas medianas y grandes. A continuación se describen las variables utilizadas en el modelo.

\section{Variables de la gestión del conocimiento aplicadas para las medianas y grandes empresas del Valle de Sugamuxi}

En este apartado se tuvieron en cuenta los postulados de Arboníes (2006), Inche \& Chung (2004), Nonaka \& Takeuchi (1995) y Pavéz (2001). Las variables aplicadas en la presente investigación se categorizaron así: - Variables de indole cualitativa. Gerentes de las empresas, capital intelectual, capital humano, capital relacional, capital estructural, área funcional investigativa, afiliación a red o asociación de II\&D, área funcional más importante, espacios de creación del conocimiento, nivel de formación académica de los gerentes, herramientas de búsqueda, comunicación y difusión del conocimiento, existencia de la empresa, tipo de vinculación de los colaboradores, nivel de formación de los colaboradores.

- Variables de indole cuantitativa. Generación de productos y/o servicios producto de la investigación, divulgación pública de productos y/o servicios, apoyo o estímulo para investigación, bases externas donde reposa la información, recursos económicos asignados a la investigación y funcionarios dedicados a II\&D.

\section{Resultados}

Con respecto a las cuarenta empresas objeto de estudio se les clasificó de acuerdo a los sectores económicos predominantes (industrial, financiero, salud, transporte y comercial) y tamaño. Se encontraron $22 \mathrm{em}$ presas grandes y 18 medianas. Los sectores más influyentes corresponden al industrial, 
financiero y transporte. El porcentaje acumulado entre estos tres sectores corresponde a un $74 \%$, mientras que salud y comercial tienen un $10 \%$ y $8 \%$, respectivamente (ver Tabla 1),

De las cuarenta empresas, trece se dedican a la prestación de servicios financieros $(33 \%)$; otra actividad que se destaca es el transporte con siete empresas (18\%) y se advierte que tres empresas son grandes y cuatro medianas (ver Tabla 2).

En cuanto al nivel de formación, el 65\% de los gerentes son especialistas y el $25 \%$ tienen magister, lo cual indica que estas organizaciones son dirigidas por ejecutivos que han tenido o se están formando a un alto nivel académico (ver Tabla 3 ).

Respecto a la relación de formación gerencial versus tamaño de las empresas, se identificó atraso académico en los niveles de alta dirección. En cuanto a estudios de maestría, solo el $27 \%$ de los directivos de grandes empresa han alcanzado este título, mientras que el $22 \%$ lo han alcanzado en las medianas empresas (ver Tabla 4).

En lo que compete a la afiliación a redes de investigación, innovación y desarrollo empresarial, se encontró que el $82,5 \%$ de las empresas estudiadas no se encuentran afiliadas, cifra que muestra la indiferencia hacia los proyectos de investigación pues únicamente siete empresas se encuentran afiliadas (ver Tabla 5).

El análisis descriptivo de los resultados se realizó con base en la escala de aspectos relevantes como: muy importante, siempre y la respuesta Sí. Los promedios de los porcentajes y de los números, que presentan las variables descritas, se dividen en intervalos, teniendo en cuenta la clasificación de los niveles correspondientes, así: de o\% al 33\% ó de o a 33 = es un nivel bajo; de $34 \%$ al $67 \%$ ó de 34 a 67 = es un nivel medio; de $68 \%$ al $100 \%$ ó de 68 a $100=$ es un nivel alto.

\subsection{Situación actual de la gestión del conoci- miento en las medianas y grandes empresas del Valle de Sugamuxi}

En este análisis se consideraron siete variables importantes dentro de las organizaciones estudiadas. En cuanto al concepto de gestión del conocimiento, el $47,5 \%$ contestó que es un proceso de creación, uso y difusión del conocimiento basado en el talento humano, coincidiendo con el concepto de Nonaka \& Takeuchi (1995). La preferencia de los gerentes está dirigida a la creación de espacios que permitan la creación, uso y difusión del conocimiento, debido a la capacitación y entrenamiento que ocupan un nivel muy alto de importancia con un $67 \%$. El desarrollo personal es un factor relevante

Tabla 1. Relación del sector económico y el tamaño empresa

\begin{tabular}{lcccccc}
\hline \multirow{2}{*}{ Sector } & \multicolumn{4}{c}{ Tamaño empresa } & \multirow{2}{*}{ Total } \\
\cline { 2 - 5 } & \multicolumn{2}{c}{ Grande } & \multicolumn{2}{c}{ Mediana } & & \\
\hline Comercial & 2 & $9 \%$ & 1 & $6 \%$ & 3 & $8 \%$ \\
Industrial & 8 & $36 \%$ & 5 & $28 \%$ & 13 & $33 \%$ \\
Financiero & 8 & $36 \%$ & 5 & $28 \%$ & 13 & $33 \%$ \\
Salud & 1 & $5 \%$ & 3 & $17 \%$ & 4 & $10 \%$ \\
Transporte & 3 & $14 \%$ & 4 & $22 \%$ & 7 & $18 \%$ \\
Total & 22 & $100 \%$ & 18 & $100 \%$ & 40 & $100 \%$ \\
\hline
\end{tabular}

Fuente: Elaboración propia. 
Caracterización y medición del nivel de gestión del conocimiento en las medianas y grandes empresas del Valle de Sugamuxi del Departamento de Boyacá

Tabla 2. Actividad que desarrolla la empresa

\begin{tabular}{|c|c|c|c|c|c|c|c|c|}
\hline \multirow{3}{*}{ Actividad } & \multirow{3}{*}{$\begin{array}{c}\mathrm{N}^{\circ} \text { de } \\
\text { empresas }\end{array}$} & \multirow{3}{*}{$\begin{array}{l}\text { Por- } \\
\text { centaje }\end{array}$} & \multirow{3}{*}{$\begin{array}{l}\text { Porcentaje } \\
\text { válido }\end{array}$} & \multirow{3}{*}{$\begin{array}{l}\text { Porcentaje } \\
\text { acumulado }\end{array}$} & \multicolumn{4}{|c|}{ Bootstrap para porcentaje } \\
\hline & & & & & \multirow[b]{2}{*}{ Sesgo } & \multirow{2}{*}{$\begin{array}{l}\text { Típ. } \\
\text { Error }\end{array}$} & \multicolumn{2}{|c|}{ IC al 95\% } \\
\hline & & & & & & & $\begin{array}{l}\text { Infe- } \\
\text { rior }\end{array}$ & $\begin{array}{c}\text { Supe- } \\
\text { rior }\end{array}$ \\
\hline $\begin{array}{l}\text { Prestación y } \\
\text { servicios finan- } \\
\text { cieros }\end{array}$ & 13 & 32,5 & 32,5 & 32,5 & 0,2 & 7,4 & 17,5 & 47,5 \\
\hline $\begin{array}{l}\text { Prestación } \\
\text { servicios de } \\
\text { Salud }\end{array}$ & 4 & 10,0 & 10,0 & 42,5 & $(0,2)$ & 4,6 & 2,5 & 20,0 \\
\hline Transportes & 7 & 17,5 & 17,5 & 60,0 & - & 5,9 & 7,5 & 30,0 \\
\hline $\begin{array}{l}\text { Comercial- } \\
\text { ización de B } \\
\text { y/o S }\end{array}$ & 5 & 12,5 & 12,5 & 72,5 & $(0,1)$ & 5,1 & 2,5 & 22,5 \\
\hline $\begin{array}{l}\text { Fundición } \\
\text { metales no fer- } \\
\text { rosos }\end{array}$ & 3 & 7,5 & 7,5 & 80,0 & 0,1 & 4,1 & - & 17,5 \\
\hline $\begin{array}{l}\text { Producción de } \\
\text { armas militares }\end{array}$ & 1 & 2,5 & 2,5 & 82,5 & 0,2 & 2,6 & - & 7,5 \\
\hline $\begin{array}{l}\text { Fabricación } \\
\text { de fertilizantes } \\
\text { fosfóricos }\end{array}$ & 1 & 2,5 & 2,5 & 85,0 & 0,1 & 2,5 & - & 10,0 \\
\hline $\begin{array}{l}\text { Producción de } \\
\text { cemento }\end{array}$ & 3 & 7,5 & 7,5 & 92,5 & $(0,1)$ & 4,1 & - & 15,0 \\
\hline Minería & 3 & 7,5 & 7,5 & 100,0 & $(0,1)$ & 4,2 & - & 17,5 \\
\hline Total & 40 & 100,0 & 100,0 & & - & - & 100,0 & 100,0 \\
\hline
\end{tabular}

Nota. a. A menos que se haga alguna anotación, los resultados del Bootstrap están basados en muestras de 1000 Bootstrap. $b$. IC=Intervalo de confianza.

Fuente: Elaboración propia.

Tabla 3. Nivel de formación del gerente

\begin{tabular}{lcccc}
\hline Nivel de formación & Empresas & Porcentaje & $\begin{array}{c}\text { Porcentaje } \\
\text { válido }\end{array}$ & $\begin{array}{c}\text { Porcentaje } \\
\text { acumulado }\end{array}$ \\
\hline Magister & 10 & 25 & 25 & 25 \\
Especialista & 26 & 65 & 65 & 90 \\
Pregrado & 4 & 10 & 10 & 100 \\
\hline Total & 40 & 100 & 100 & \\
\hline
\end{tabular}

Fuente: Elaboración propia. 
José Javier González Millán, Miryam Teresa Rodríguez Díaz

\& Edith Viviana Cárdenas Barrera

Tabla 4. Tamaño empresa: nivel formación del gerente

\begin{tabular}{lcccc}
\hline \multirow{2}{*}{ Tamaño empresa } & \multicolumn{3}{c}{ Nivel de formación } & \multirow{2}{*}{ Total } \\
\cline { 2 - 4 } & Magister & Especialista & Pregrado & \\
\hline Grande & 6 & 13 & 3 & 22 \\
Mediana & 4 & 13 & 1 & 18 \\
\hline Total & 10 & 26 & 4 & 40 \\
\hline
\end{tabular}

Fuente: Elaboración propia.

Tabla 5. Red de asociación de investigación, innovación y desarrollo

\begin{tabular}{|c|c|c|c|c|}
\hline Red & Empresas & Porcentaje & $\begin{array}{l}\text { Porcentaje } \\
\text { válido }\end{array}$ & $\begin{array}{l}\text { Porcentaje } \\
\text { acumulado }\end{array}$ \\
\hline Inexistencia & 33 & 82,5 & 82,5 & 82,5 \\
\hline ASEFINSOL & 1 & 2,5 & 2,5 & 85,0 \\
\hline $\begin{array}{l}\text { ASEMI (Asociación Colombiana de } \\
\text { Empresas de Medicina Integral) }\end{array}$ & 1 & 2,5 & 2,5 & 87,5 \\
\hline $\begin{array}{l}\text { Asofinso (Asociación de Entidades } \\
\text { Financieras de Sogamoso) }\end{array}$ & 1 & 2,5 & 2,5 & 90,0 \\
\hline $\begin{array}{l}\text { ASOTRANS (Asociación Nacional de } \\
\text { Transporte) }\end{array}$ & 1 & 2,5 & 2,5 & 92,5 \\
\hline $\begin{array}{l}\text { CIDET (Centro de Investigación y } \\
\text { Desarrollo Tecnológico del Sector } \\
\text { Eléctrico) }\end{array}$ & 1 & 2,5 & 2,5 & 95,0 \\
\hline COLFECAR & 1 & 2,5 & 2,5 & 97,5 \\
\hline SABMILLER & 1 & 2,5 & 2,5 & 100,0 \\
\hline Total & 40 & 100 & 100 & \\
\hline
\end{tabular}

Fuente: Elaboración propia.

que permite la difusión del conocimiento, ya que presentan un nivel de preferencia muy importante con un $37,5 \%$. Los escenarios tecnológicos para gestionar el conocimiento mediante software especializados presenta un $38,7 \%$, este es el nivel más importante, lo que permite concluir que las organizaciones prefieren gestionar el conocimiento invirtiendo en tecnología.

Las formas para identificar, guardar y re-utilizar la información corresponden al uso de la memoria magnética que equivale al 55\%. Los sistemas para codificar, transferir y capitalizar los flujos de conocimiento no son tenidos en cuenta. Esto evidencia que las empresas no tienen la capacidad para manejar sistemas sofisticados con el conocimiento que poseen. La permanencia del conocimiento cuando alguien se retira de la organización presenta un $71 \%$.

Así mismo, se concluyó que siempre ha existido la evaluación del desempeño para la retroalimentación del conocimiento pues la aplicación de programas de inducción para 
todos los colaboradores de la organización presentó un resultado positivo con un $95 \%$. Las barreras que impiden el desarrollo de la gestión del conocimiento presentan un $27,5 \%$, debido al apego a normas y procedimientos pre-elaborados. Paralelamente, el $22,5 \%$, corresponde a la falta de estímulos a los colaboradores dentro de la organización respecto a la innovación y la creatividad.

En resumen, la situación actual de la gestión del conocimiento en las medianas y grandes empresas del Valle de Sugamuxi presenta un nivel de desarrollo del conocimiento medio. En la Tabla 6 se hace un resumen en donde se destacan los elementos más representativos de cada uno de los ítems expuestos en este apartado.

Con base en la Tabla 6 es de destacar que los elementos más representativos son las practicas, tanto al interior de la organización (92,5\%) como de formación, capacitación, educación y desarrollo con un 95\%; sin embargo, hay una serie de variables criticas como los sistemas de codificación y transferencia de conocimiento, que pasan al anonimato perjudicando altamente la difusión del conocimiento organizacional.

\subsection{Diagnóstico de los procesos de la gestión del conocimiento en las medianas y grandes empresas del Valle de Sugamuxi}

De acuerdo con el ciclo de conocimiento de Nonaka \& Takeuchi (1995), se encontró que los aspectos más importantes, presentados en cada fase de los procesos de socialización, exteriorización, combinación e interiorización, son los siguientes:

Proceso de socialización de conocimiento en las medianas y grandes empresas del Valle de Sugamuxi

En esta fase se logra el proceso de conversión del conocimiento de tácito a explícito, para lo cual se tuvieron en cuenta doce (12) ítems, de los cuales se presentan los factores más representativos para cada ciclo de la gestión del conocimiento, a saber:

Para permitir la correcta socialización del conocimiento los gerentes de las empresas del Valle de Sugamuxi, afirman que la variable que se usa con más frecuencia es la acción de elaboración de manuales, representado con el $70 \%$; se identificó que del total de las 22 empresas grandes, 17 realiza esta acción y de las 18 medianas, 11 llevan a cabo este proceso.

Otro aspecto por resaltar es la elaboración de documentos con una participación del 42,5\%, lo cual indica que el método más utilizado para compartir un conocimiento es el escrito.

Por último, el aprendizaje en equipo es relevante como acción de socialización, se cuenta con un 32,5\% de participación, seguido de la socialización de experiencias y habilidades con el $30 \%$.

Proceso de exteriorización de conocimiento en las medianas y grandes empresas del Valle de Sugamuxi

Alude a la conversión de conocimiento tácito a explícito, donde el análisis presenta nueve (9) ítems, de los cuales la investigación muestra las variables más utilizadas por las organizaciones del Valle de Sugamuxi.

En este proceso se identificó que las empresas, por lo general, no consideran importante el uso de las prácticas. Sin embargo, se encuentran variables más significativas, como lo son los conceptos de producción que presentan un $32,5 \%$; también existen dentro de la organización la capacidad para identificar, estandarizar y transferir practicas de gestión del conocimiento con un $22 \%$, las posibilidades de acceder a nuevos conocimientos para la comunidad y el uso de slogan tienen un $22 \%$. La utilización de modelos presenta un $15 \%$ lo cual indica que 
José Javier González Millán, Miryam Teresa Rodríguez Díaz

\& Edith Viviana Cárdenas Barrera

Tabla 6. Promedio de la situación actual de la gestión del conocimiento

\begin{tabular}{lc}
\hline Descripción & Porcentaje \\
\hline Concepto de gestión del conocimiento & 47,5
\end{tabular}

La gestión del conocimiento, es un proceso de creación, uso y difusión del cono-

cimiento basado en el talento humano

Espacios que permiten la creación, uso y difusión del conocimiento

capacitación y entrenamiento espacios de creación, uso y difusión del conocimiento

Factor relevante que permite la difusión del conocimiento

Desarrollo personal

Escenarios tecnológicos para gestionar el conocimiento

Software especializado para gestionar el conocimiento

Formas para identificar, guardar y reutilizar el conocimiento

Utilización de la memoria magnética para identificar, guardar y reutilizar inequívocamente la información relevante de la organización

Sistemas para codificar, transferir y capitalizar los flujos de conocimiento

No poseen

Permanencia del conocimiento en la organización cuando alguien se retira de la organización

Evaluación del desempeño para la retroalimentación del conocimiento

Prácticas que se llevan a cabo al interior de la organización

La formación de los colaboradores dentro de la organización influye en el desarrollo de la gestión del conocimiento

Prácticas de educación, capacitación, formación y desarrollo que se aplican en la organización

Aplicación de programas de inducción para todos los colaboradores

*Barreras que impiden el desarrollo de la Gestión del Conocimiento en la organización

Apegado a las normas y procedimientos pre elaborados

$\begin{array}{lr}\text { Promedio } & 56 \% \\ \text { Nivel catalogado } & \text { Medio }\end{array}$

Nota. ${ }^{\star}$ Las barreras de la Gestión del Conocimiento son inversas por lo tanto no se tiene en cuenta. Fuente: Elaboración propia.

se tienen acciones de exteriorización en un nivel de importancia bajo.

Proceso de combinación de conocimiento en las medianas y grandes empresas del Valle de Sugamuxi

Esta fase se refiere a la conversión de conocimiento explícito a implícito, para lo cual se trabajó con cinco (5) factores de análisis, identificando que para estas organizaciones la variable más significativa es el uso de e-mails.

El estudio muestra que el $67,5 \%$ de las empresas siempre hacen uso de e-mails, como herramienta fundamental para la comunicación cotidiana debido al auge que ha tenido el internet. Oros aspectos importantes son las comunicaciones como el correo físico, que 
tiene un $57,5 \%$, las conversaciones telefónicas con un 52,5\%. Los resúmenes investigativos presentan un nivel bajo de importancia con un $15 \%$. Por lo tanto, el estilo de las organizaciones, para realizar las acciones de combinación de conocimiento, es relativamente favorable aunque un poco tradicionales.

Proceso de interiorización de conocimiento en las medianas y grandes empresas del Valle de Sugamuxi

Esta última fase hace referencia a la conversión de conocimiento de explícito a tácito. La investigación demuestra que el proceso de interiorización del conocimiento más significativo en las medianas y grandes empresas del Valle de Sugamuxi es la acción que permite que las experiencias, procesos y procedimientos aplicados se almacenen en memorias, documentales u otros medios. Se obtiene un porcentaje total del $55 \%$ en el nivel más alto, teniendo en cuenta que de las 22 grandes empresas, 14 siempre realizan esta práctica. Otra acción de interiorización es la incorporación del conocimiento a bases de datos con una participación del $27,5 \%$, mientras que el uso de modelos mentales tienen una participación del 12,5\% y las prácticas de trabajo investigativo presentan un $7,5 \%$. Estas acciones de interiorización permiten afirmar que las organizaciones presentan un nivel medio no favorable para el desarrollo de la gestión del conocimiento en estos aspectos. En la Tabla 7 se pueden visualizar los aspectos pertinentes en cada una de las fases nombradas en este apartado.

La Tabla 7 muestra los factores más relevantes del ciclo de creación de conocimiento, destacando el uso de e-mail, correos físicos, conversaciones telefónicas, prácticas de trabajos investigativos y la socialización mediante la elaboración de manuales, documentos, aprendizaje en equipo y la compartición de experiencias y habilidades.

Lo antes expuesto demuestra que el diagnóstico de los procesos de la gestión del conocimiento en las medianas y grandes empresas del Valle de Sugamuxi tiene un

Tabla 7. Ciclo del conocimiento de Nonaka y Takeuchi en las medianas y grandes empresas del Valle de Sugamuxi

Tácito a explícito

\begin{tabular}{|c|c|}
\hline $\begin{array}{l}\text { Proceso de socialización }(43,8 \%) \\
\text { - Elaboración de manuales } \\
\text { - Elaboración de documentos } \\
\text { - Aprendizaje en equipo } \\
\text { - Compartición de experiencias y } \\
\text { habilidades }\end{array}$ & $\begin{array}{l}\text { Proceso de eteriorización (23\%) } \\
\text { - Conceptos de producción } \\
\text { - Capacidad para identificar, es- } \\
\text { tandarizar y transferir prácticas de } \\
\text { gestión del conocimiento } \\
\text { - Acceso a conocimientos para la } \\
\text { comunidad }\end{array}$ \\
\hline $\begin{array}{l}\text { Proceso de interiorización }(25,6 \%) \\
\text { - Almacenamiento en memorias do- } \\
\text { cumentales las experiencias, procesos } \\
\text { y procedimientos aplicados } \\
\text { - Incorporación del conocimiento a } \\
\text { bases de datos de conocimiento } \\
\text { - Uso de modelos mentales } \\
\text { - Trabajo en equipo }\end{array}$ & $\begin{array}{l}\text { Proceso de combinación (48\%) } \\
\text { - Uso de e-mail } \\
\text { - Correos físicos } \\
\text { - Conversaciones telefónicas } \\
\text { - Resúmenes investigativos }\end{array}$ \\
\hline
\end{tabular}


nivel de desarrollo medio ya que, tomando la frecuencia mayor en cuanto al nivel alto, medio y bajo encontrados, se tienen los resultados de la Tabla 8.

\subsection{Factores relevantes de la gestión del} conocimiento en las medianas y grandes empresas del Valle de Sugamuxi

Factores organizacionales de orden cualitativo

Para este apartado se tuvieron en cuenta diez aspectos que p ermiten la identificación de estos factores en las medianas y grandes empresas del Valle de Sugamuxi, en relación a la gestión del conocimiento. En estos, las capacidades del gerente para identificar, valorar y desarrollar son equivalentes a las capacidades de investigación, innovación y de creatividad con un $27,6 \%$, lo cual demuestra que sí existen posibilidades para desarrollar la gestión del conocimiento apropiadamente, ya que se requiere de una actitud investigativa y de los conocimientos necesarios para poder desarrollar esta actividad. Los

Tabla 8. Promedio del diagnóstico de los procesos de la gestión del conocimiento

\begin{tabular}{lc}
\hline Descripción & Porcentaje \\
\hline Proceso de socialización & 47,5 \\
Elaboración de manuales & \\
Elaboración de documentos & \\
Aprendizaje en equipo & 67 \\
Compartición de experiencias y habilidades & \\
Proceso de exteriorización & \\
Conceptos de producción & \\
Capacidad para identificar, estandarizar y transferir practicas de gestión del conocimiento & \\
Acceso a conocimientos para la comunidad & 37,5 \\
Uso de slogan & \\
Utilización de modelos & \\
Proceso de combinación & \\
Uso de E-mail & \\
Correos físicos & \\
Conversaciones telefónicas & \\
Resúmenes investigativos & \\
Proceso de interiorización & \\
Almacenamiento en memorias documentales las experiencias, procesos y procedimien- \\
tos aplicados \\
Incorporación del conocimiento a bases de datos de conocimiento \\
Uso de modelos mentales \\
Trabajo en equipo & \\
\hline Promedio & \\
Nivel catalogado & \\
\hline
\end{tabular}


conceptos de capital intelectual en un 15,2\% de las veces se valoran como buenos y en un $39,4 \%$ como regulares o malos, lo cual indica que verdaderamente cinco de los gerentes de las empresas grandes respondieron bajo el desconocimiento de la Norma. En cuanto al capital relacional, se valoraron como buenos en un $32,1 \%$ de las veces, como regulares en un $46,4 \%$ y como malo en un $17,9 \%$; esto demuestra que este concepto es más conocido que el capital intelectual pero no lo suficiente.

Para el capital estructural, los conceptos de los gerentes son buenos el $39,1 \%$ de las veces, $36,1 \%$ son regulares y el $30,4 \%$ malos, demostrando que este concepto es más conocido en las grandes empresas, pero aún así no es un nivel óptimo para el desarrollo de la gestión del conocimiento. Por su parte, el capital humano está en la categoría de conceptos buenos con un $46,2 \%$; el $43,6 \%$ son regulares y el $7,7 \%$ son malos, por lo que se concluye que este concepto es más conocido en las grandes empresas, lo cual es favorable para el desarrollo de la gestión del conocimiento.

En cuanto al área funcional de las organizaciones que realiza las investigaciones e innovaciones, el $45 \%$ no tiene un área funcional que realice las investigaciones e innovaciones, el $55 \%$ está distribuido en varias áreas, el 22,5\%, hace referencia al área de investigación y desarrollo; el 12,5\% del área administrativa es quien realiza la investigaciones; el 10\% pertenece al área de control de calidad y el $5 \%$ para las áreas de mercadeo y talento humano. Lo anterior demuestra que sí existen las posibilidades para fortalecer el área de la investigación favorablemente en las organizaciones del Valle de Sugamuxi.

Estas organizaciones en un $82,5 \%$, no se encuentran afiliada a una red o asociación de II\&D, a pesar que esto permite generar nuevos conocimientos o descubrimientos que aportan valor a las organizaciones. Sin embargo, esta cifra muestra la indiferencia en los proyectos de investigación que pueden ser útiles, en donde el área funcional más importante es la del talento humano en un $60 \%$, aunque este es un porcentaje a nivel medio, lo cual significa que los colaboradores son medianamente importantes dentro de las organizaciones.

Otras áreas importantes son la de producción con un $15 \%$, seguida de la financiera y marketing con un $10 \%$, mostrando que el valor del las personas esta por encima de otras áreas. El medio de difusión del conocimiento más utilizado en la organización es la publicación electrónica con una participación del $30 \%$, esto indica que los gerentes optan por difundir el conocimiento haciendo uso de nuevas tendencias tecnológicas, sin dejar a un lado otros medios como el texto escrito y las exposiciones magistrales.

Se encontró que el $65 \%$ de los gerentes tienen nivel de formación de especialista, un $25 \%$ de magister y el $10 \%$ hasta pregrado, lo cual indica que estas organizaciones son dirigidas por funcionarios que han tenido $o$ se están formando académicamente; en el nivel de formación de los colaboradores el más alto es el de especialista (62,5\%), por lo tanto, corresponde a medio. $\mathrm{Al}$ respecto, se considera que para el desarrollo de la gestión del conocimiento es indispensable conocer nuevas tendencias administrativas.

Dentro de la medianas y grandes empresas del Valle de Sugamuxi, el uso de Internet como herramienta de búsqueda y transmisión del conocimiento se midió por medio de franjas en horas semanales. Los resultados muestran que el $35 \%$ de los encuestados se encuentra en el rango de 16 a 25 horas, el 20\% en la franja de 5 a 8 horas, el $17 \%$ en la franja de o a 1 hora, el $15 \%$ en la franja de 2 a 4 horas, el 12,5\% en la franja de nueve a 15 horas.

Por otro lado, es importante tener en cuenta el tiempo de existencia de la empresa. 
El 70\% de las organizaciones tiene más de 21 años de existencia, el $12,5 \%$ tiene un tiempo de 11 a 15 años, el $10 \%$ tiene hasta cinco años de existencia y el $7,5 \%$ tiene de seis a diez años de existencia. Se concluye que el $30 \%$ de las empresas son relativamente jóvenes, lo cual puede predecir un favorable a futuro del desarrollo de la gestión del conocimiento.

En cuanto al tipo de contratación de los colaboradores, el personal más vinculado es a término fijo, hecho que corresponde al $77,5 \%$, seguido de los empleados a término indefinido con un $22,5 \%$. Los factores relevantes de la gestión del conocimiento de orden cualitativo presentan un nivel de desarrollo medio en las medianas y grandes empresas del Valle de Sugamuxi, ya que los intervalos estipulados para este nivel oscilan entre el 34\% al $67 \%$ y el número de mayor frecuencia se encuentra en nivel medio discriminándose como en la Tabla 9.

De acuerdo con la Tabla 9 se puede afirmar que los factores de mayor influencia son el tipo de contratación $(77,5 \%)$ y el nivel de formación con un $65 \%$, lo cual permite establecer que cualitativamente son dos factores que inciden en las políticas de desarrollo de personal al interior de las organizaciones.

Factores organizacionales de orden cuantitativo

El 85\% de las empresas muestran que no hay ni creación ni innovación en cuanto a productos y/o servicios. $\mathrm{El}$ 50\% de las empresas

Tabla 9. Factores relevantes de orden cualitativo

\begin{tabular}{lc}
\hline Descripción & Porcentaje \\
\hline Capacidad del gerente para identificar, valorar y desarrollar la gestión del conocimiento & \\
Capacidad investigativa, innovación y de creatividad & 27,6 \\
Capital intelectual (conceptos en la categoría de buenos) & 15,2 \\
Capital relacional (conceptos en la categoría de buenos) & 32,1 \\
Capital estructural (conceptos en la categoría de buenos) & 39,1 \\
Capital humano (conceptos en la categoría de buenos) & 46,2 \\
Área funcional de la organización que realiza las investigaciones (investigación y & \\
desarrollo) & 55,0 \\
Afiliación a un red o asociación de II\&D & 17,5 \\
Área funcional más importante (Área de Talento Humano) & 60,0 \\
Medio de difusión del conocimiento más utilizado en la organización & \\
Publicaciones electrónicas & 30,0 \\
Nivel de formación de los gerentes (Especialistas) & 65,0 \\
Nivel de formación de los colaboradores (Especialistas) & 62,5 \\
El uso de Internet como herramienta de búsqueda y transmisión del conocimiento & \\
(de 16 a 25 horas semanales) & 35,0 \\
Tiempo de existencia de la empresa (21 años en adelante) & 12,5 \\
Tipo de contratación de los colaboradores (término fijo) & 77,5 \\
\hline Promedio & $41 \%$ \\
Nivel catalogado & Medio \\
\hline
\end{tabular}


presenta nuevas creaciones en servicios, el $33,3 \%$, realiza innovaciones en productos y el $16,7 \%$ realiza nuevas creaciones en servicios; lo anterior demuestra que este factor presenta un nivel significativamente bajo. De las seis empresas que presentaron creación e innovación, dos de estas innovaron en productos como fertilizantes fosfóricos (Fosfatos de Boyacá) y herrajes plásticos (Fundi Herrajes); la empresa que innovó en servicios corresponde a la Banca Móvil Cerca (AVillas) y las tres que crearon nuevos productos fueron el Centro de Control Integral (actualización del sistema eléctrico EBSA), Control de Cargue de Mercancías (Transboy) y la Plazoleta de Comidas (La Canasta), con un total de seis tipos de generaciones de productos y/o servicios.

En cuanto al apoyo o estímulo para la investigación, se determinó que el número de apoyos dados a los funcionarios como corresponde: los transportes a eventos fueron 9, inscripción eventos 6; estudios avanzados y estímulo económico 5; apoyo de gastos de viajes 4; adquisición de literatura 2; apoyos artísticos y de patentes 1; esto permitió identificar que el apoyo a trasportes es el más importante para estas organizaciones, con un total de 36 estímulos otorgados por año a los funcionarios de las organizaciones.

En cuanto a la divulgación pública de la generación de productos y/o servicios, los empresarios del Valle de Sugamuxi no poseen la cultura de hacer investigación en sus organizaciones, por lo tanto, no brindan a los colaboradores ni a la sociedad la posibilidad de divulgación de productos y/o servicios fruto de la investigación, luego, el rango de divulgaciones oscila tan solo entre 1 a 3, donde los capítulos de libros, literatura gris y artículos tienen una sola divulgación; los artículos de investigación, tesis, normas, productos no patentados, tienen dos divulgaciones y otras publicaciones tienen tres; el número de divulgaciones en total es 16 , lo cual presenta un nivel muy bajo;

Respecto a las bases externas donde reposa la información, estas no son empleadas por el 55\% de los encuestados; sin embargo, el $45 \%$ cuenta con una sola base externa de investigación, dos empresas cuentan con dos bases externas y una cuenta con tres; esto muestra que existe un nivel bajo de información de bases externas.

En cuanto a la asignación presupuestal para la investigación, innovación y desarrollo, solo dos hicieron mención del valor que se invierte para esta actividad anualmente, COP\$35.000.000 para la empresa SANOHA y COP\$50.000.000, para La Clínica de Especialistas. Debido a que esta información es reservada, pues los gerentes de las organizaciones son muy renuentes a compartir los rubros de su presupuesto, solo se pudo conocer el valor de estas organizaciones; $\sin$ embargo, 22 organizaciones realizan labores de investigación y, de acuerdo con el número de funcionarios dedicados a esta labor, se encuentra que: el 55\% de las organizaciones no posee funcionarios dedicados a esta labor, el 22,5\% de las empresas cuentan con uno, el $15 \%$ tienen dos funcionarios y el 2,5\% tienen de once a quince. Se puede concluir entonces que las medianas y grandes empresas del Valle de Sugamuxi no poseen un número considerable de colaboradores que se dediquen a la investigación y creatividad.

En cuanto a los factores relevantes de la gestión del conocimiento de orden cuantitativo, predomina un nivel de desarrollo bajo, teniendo en cuenta los intervalos estipulados para este nivel de o a 33 (ver Tabla 10). Se deduce que estos factores son muy importantes porque constituyen algunas de las variables independientes que influyen en la generación de productos y/o servicios que es la variable dependiente, la cual se decidió tomar ya que el desarrollo de la gestión del 
Tabla 10. Factores relevantes de orden cuantitativo

\begin{tabular}{lc}
\hline Descripción & Número \\
\hline Generación de productos y/o servicios & 6 \\
Apoyo o estímulos para la investigación & 36 \\
Divulgación pública de productos y/o servicios & 16 \\
Número de bases externas donde reposa la información & 21 \\
Asignación presupuestal para la investigación, innovación y desarrollo & 2 \\
Número de funcionarios que se dedican a realizar investigación & 48 \\
\hline Promedio & 22 \\
Nivel catalogado & Bajo \\
\hline
\end{tabular}

Fuente: Elaboración propia.

conocimiento permite la creación e innovación en una organización. Para observar con más detalle la influencia de estas variables se realiza un análisis de regresión ANOva y factorial.

En lo concerniente al orden cuantitativo, la relación es muy pobre, toda vez que como se aprecia en la Tabla 10, la inversión económica es muy baja, demostrando cómo en algunas compañías la investigación e innovación no son factores relevantes en la empresa. Igualmente, es muy bajo el número de funcionarios que se dedican a estas labores y esto repercute en la baja generación de productos y servicios de los sectores empresariales del Valle de Sugamuxi.

\subsection{Análisis factorial}

Para el desarrollo de este apartado se acudió al método de Factores Comunes propuesto por Díaz (2002); además, se realizó un análisis de ANOva que hace referencia a la correlación y al grado de significancia de las variables consideradas más importantes con el propósito de analizarlas y observar el impacto que éstas tienen sobre la variable dependiente. Para lograr este objetivo se cruzó la variable dependiente número de productos y/o servicios, con cada una de las siete variables independientes estipuladas según De los Santos (1997), Vargas \& Moreno (2002) y González (2009), que son: número de bases externas donde reposa la información de la organización, nivel de formación de los colaboradores, uso de horas de internet, tiempo de existencia de la organización, número de colaboradores que están dedicados a la investigación, empleados a término fijo y empleados a término indefinido.

$\mathrm{El}$ análisis de factores comunes por el método de componentes principales, corresponde al estudio en donde ya se ha seleccionado o decantado una serie de variables incidentes en una dependiente, de las cuales solamente se aceptaron dos por su nivel de correlación: los empleados a término fijo y los empleados a término indefinido. A continuación, se presentan las estadísticas descriptivas del análisis factorial para llegar a la depuración final de factores comunes que explicarán la variable independiente que influye en la dependiente, tal como se puede observar en la Tabla 11.

La variable que explica el desarrollo de la generación de productos y/o servicios es la de los colaboradores que son contratados a término fijo, logrando así explicar la varianza en un $76,5 \%$, esto quiere decir que la contratación a término fijo es la variable más cercana al origen del eje Y (ver Gráfico 
Tabla 11. Varianza total explicada*

\begin{tabular}{ccccccc}
\hline & \multicolumn{5}{c}{ Componente } \\
\cline { 2 - 7 } Dimensión & \multicolumn{3}{c}{ Autovalores iniciales } & \multicolumn{3}{c}{$\begin{array}{c}\text { Sumas de las saturaciones al } \\
\text { cuadrado de la extracción }\end{array}$} \\
\cline { 2 - 7 } & Total & $\begin{array}{c}\text { \% de la } \\
\text { varianza }\end{array}$ & $\%$ acumulado & Total & \% de la varianza & $\%$ acumulado \\
\hline 1 & 1 & 39,5 & 39,5 & 1 & 39,5 & 39,5 \\
2 & 1 & 37,0 & 76,5 & 1 & 37,0 & 76,5 \\
3 & 0 & 23,4 & 100,0 & & & \\
\hline
\end{tabular}

Nota. ${ }^{\star}$ Método de extracción: Análisis de Componentes principales.

Fuente: Elaboración propia.

Gráfico 2. Componentes principales del análisis factorial

Gráfico de componentes

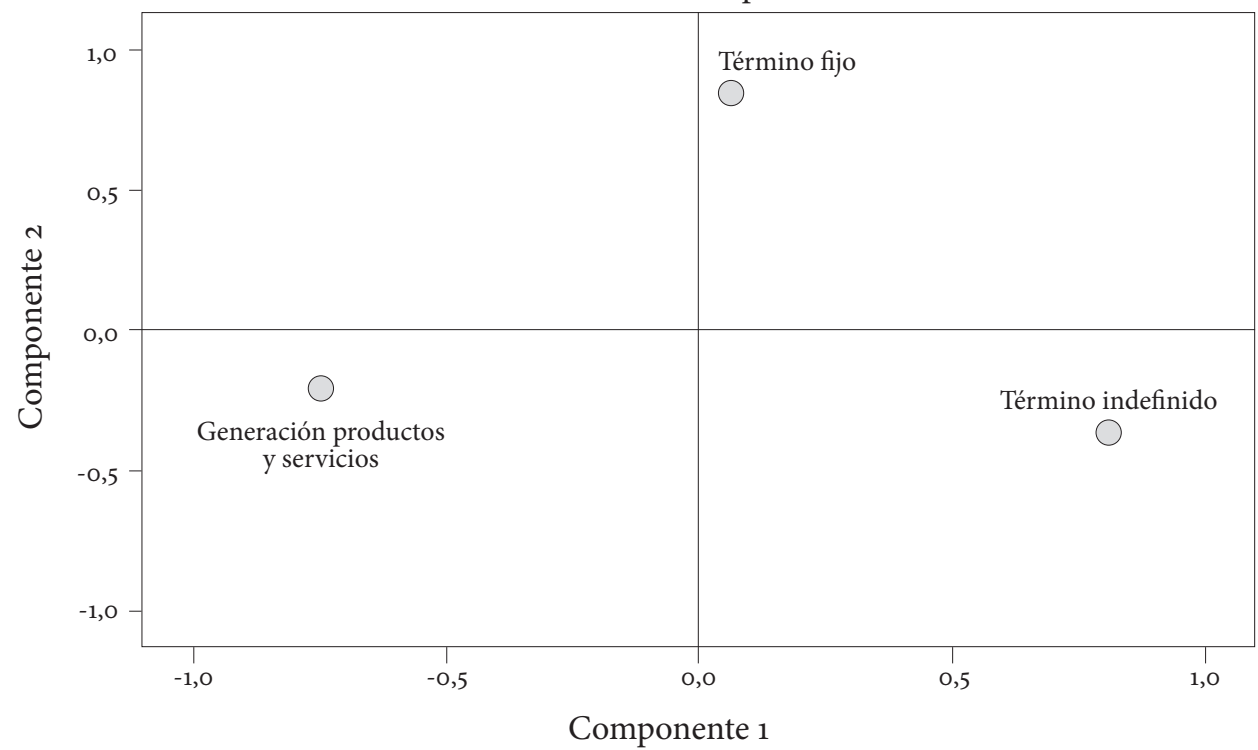

Fuente: Elaboración propia.

2). Esta variable influye favorablemente en la generación productos y/o servicios, como capital productivo en las organizaciones estudiadas, ya que las personas, al conocer el tiempo exacto en que transcurrirán sus actividades, tienen la oportunidad de ser más productivas y generar ideas nuevas y creativas dentro de las medianas y grandes empresas del Valle de Sugamuxi.
3.5 Modelo teórico de gestión del conocimiento para las medianas y grandes empresas del Valle de Sugamuxi

El modelo de gestión propuesto para las medianas y grandes empresas del Valle de Sugamuxi (ver Gráfico 3) se basa en las postulaciones de Arboníes (2006) que permiten llevar a cabo el proceso de creación adecuado; así como el uso y difusión del conocimiento en la organización, recono- 
Gráfico 3. Modelo de gestión de conocimiento para las medianas y grandes empresas del Valle de Sugamuxi

Modelo de gestión para la medianas y grandes empresas del Valle de Sugamuxi
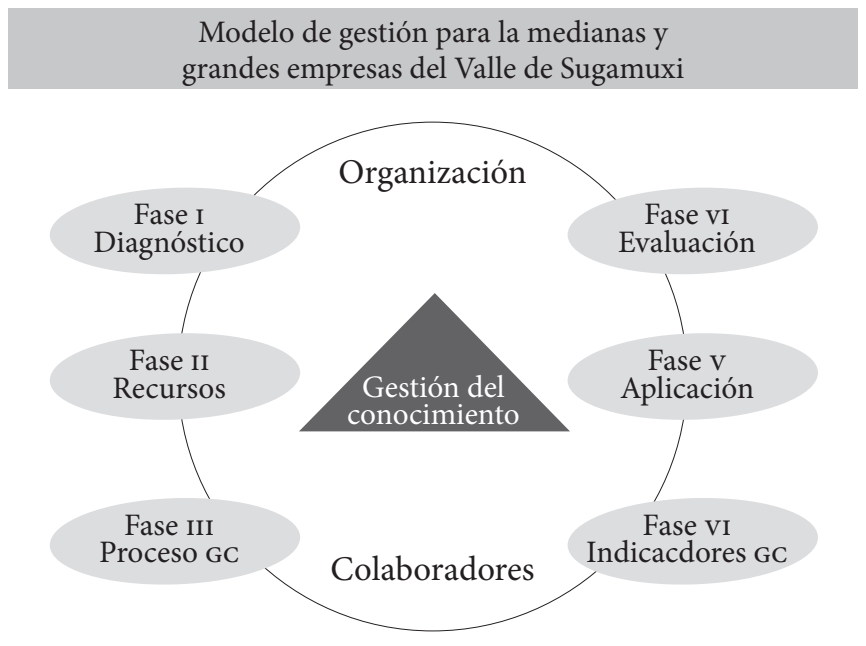

Fuente: Elaboración propia.

ciendo además que los gerentes y colaboradores influyen en el proceso de la gestión del conocimiento.

El modelo se soporta en las generaciones de conocimiento descritas por Arbonies (2006), en donde los cuatro primeros tipos de generación se relacionan con las cuatro fases del modelo propuesto, esto permite una aproximación hacia un nivel óptimo de desarrollo dentro de las organizaciones. Dicho modelo presenta seis fases, resumidas en la Tabla 12.

\subsection{Condiciones para aplicar el modelo de} gestión propuesto

A continuación se muestra el grupo de condiciones mínimas para la aplicación del modelo de gestión por parte de los gerentes y empresarios de las medianas y grandes empresas del Valle de Sugamuxi, esto gracias a los resultados obtenidos en el desarrollo de la investigación:

- Ser conscientes de la importancia de la implementación de la gestión del conocimiento en las organizaciones para permanecer dentro del mercado a futuro.
- Estar dispuestos a invertir en activos intangibles para el buen funcionamiento de la organización.

- Disponer de una formación adecuada para adquirir habilidades y capacidades de desarrollo de la gestión del conocimiento dentro de las organizaciones.

- Crear espacios de creación cultural dentro de la organización, ambientes fundamentales en donde los colaboradores pueden expresar sus ideas y contribuir a la creatividad e intercambio del conocimiento.

- Mantener, dentro de la organización, modelos que permitan la medición constante del desarrollo de la gestión del conocimiento.

\section{Conclusiones}

De acuerdo con la investigación realizada, se concluye indiscutiblemente que es el proceso de socialización y de combinación de conocimiento, los que se presentan con mayor frecuencia al interior de las medianas y grandes empresas, hecho que tiene validez en la facilidad de compartir el conocimiento de los empleados en torno al aprendizaje en equipo. Ahora bien, con base en el proceso 
Tabla 12. Las seis fases del modelo de gestión de conocimiento

\begin{tabular}{ll}
\hline Fase & Definición \\
\hline Primera fase: Diagnóstico & $\begin{array}{l}\text { Situación actual de la empresa, detecta las barreras organizacionales, } \\
\text { planea objetivos y genera estrategias. } \\
\text { Segunda fase: Recursos } \\
\text { Se refiere a los recursos tangibles e intangibles (capital humano, capital } \\
\text { relacional, capital estructural y capital intelectual). }\end{array}$ \\
$\begin{array}{l}\text { Tercera fase: Procesos de } \\
\text { gestión del conocimiento }\end{array}$ & $\begin{array}{l}\text { Son los procesos propuestos por Nonaka y Takeuchi, socialización, } \\
\text { exteriorización, combinación e interiorización. }\end{array}$ \\
$\begin{array}{l}\text { Cuarta fase: Indicadores } \\
\text { de gestión }\end{array}$ & $\begin{array}{l}\text { Los indicadores propuestos poseen cualidades de importancia, en } \\
\text { cuanto a la razonabilidad, de lo que se desea; facilidad, para obtener } \\
\text { los datos y generalidad, que significa que estos indicadores pueden ser } \\
\text { aplicados por cualquier organización, sin importar el tamaño y el sec- } \\
\text { tor al que pertenezca. }\end{array}$ \\
Quinta fase: Aplicación & $\begin{array}{l}\text { Tiene por objetivo la ejecución del modelo, el fin de identificar el im- } \\
\text { pacto de la gestión del conocimiento, respecto al nivel de desarrollo de } \\
\text { la organización. } \\
\text { Sexta fase: Evaluación }\end{array}$ \\
$\begin{array}{l}\text { Analiza los resultados obtenidos durante la ejecución del modelo, y de } \\
\text { acuerdo a esto realizar la retroalimentación y seguimiento de cada fase. }\end{array}$
\end{tabular}

Fuente: Elaboración propia.

de combinación, las organizaciones objeto de investigación convierten el conocimiento explicito en conceptos explícitos, a través del uso de herramientas tecnológicas, como la internet, ante lo cual cobran validez los medios de difusión y compartición de conocimiento.

Gracias a una revisión detallada de ciertos constructos epistemológicos, encausados por un camino investigativo serio y riguroso, se pudo conocer el estado actual de la gestión del conocimiento en las medianas y grandes empresas del Valle de Sugamuxi, el cual es relativamente bajo. Así mismo, es pertinente aclarar que hay un desconocimiento, por parte de los gerentes, en cuanto a la gestión del conocimiento, esto fundamentado en el hecho que, en estas organizaciones, no existe un proceso de medición de activos intangibles, ya que no emplean indicadores de modelos de gestión adecuados que les permita mejorar el desempeño organizacional y mucho menos empresarial.
Con respecto a las barreras de la gestión del conocimiento, en las empresas del Valle de Sugamuxi se hace evidente que existen tendencias tradicionales en la gerencia que se centran en el exceso de normativismo y procesos pre elaborados con respecto al quehacer cotidiano. Igualmente, la carencia de factores motivacionales para los empleados de las organizaciones que generen estimulo al interior de las compañías, se convierte en obstáculo para su pleno desarrollo (gestión del conocimiento) en un momento histórico en el cual se requiere desarrollo del capital humano.

Adicionalmente, existen otros factores que afectan una optima aplicación de la gestión del conocimiento. Dentro de estos se encuentra el nivel de formación de los gerentes, puesto que esta hace que la alineación estratégica difiera sensiblemente en torno al quehacer de las áreas funcionales de las compañías, encontrándose que estas organizaciones, por lo general, no tienen área funcional que se dedique a actividades 
fundamentales como la investigación, la innovación y el desarrollo empresarial; situación que impide la creación e innovación en productos y/o servicios.

En lo concerniente al nivel de desarrollo de estas organizaciones, se considera que es medio y que requiere de atención para un óptimo desarrollo en el futuro. En cuanto a los factores relevantes de la gestión del conocimiento de orden cuantitativo, se encontró que es bajo debido a la escasa generación de productos y/o servicios. Se encuentra que el impacto que ha tenido la gestión del conocimiento es mínimo en términos cuantificables, lo cual demuestra un marcado desinterés, por parte de los gerentes de las empresas, para gestionar el conocimiento en las organizaciones estudiadas.

En lo concerniente al análisis factorial, la extracción de componentes principales permitió concluir que la generación de productos y/o servicios está estrechamente relacionada con los contratos de los colaboradores en un porcentaje del $76,5 \%$, lo cual quiere decir que las condiciones de mejora laboral son un factor altamente incidente en la gestión del conocimiento de dichas entidades, proyectándose como una forma de estimular la creación, la innovación y la generación de nuevos productos y servicios al interior de la empresas de la región.

La presente investigación se afianza como una guía para estudios venideros, puesto que en el Departamento de Boyacá es el primero en su área, al igual que para la Universidad Pedagógica y Tecnológica de Colombia es el primero en este género, lo que a nivel de la academia permitirá que sea un referente teórico-conceptual para dar pie a otros procesos de profundización en torno al tema de la gestión del conocimiento. Esto permitirá una mayor rigurosidad en futuras investigaciones que se susciten en otras regionales del país y para otras instituciones de educación que trabajen esta línea de investigación, buscando afianzar postulados universales y más pertinentes a cada objeto de estudio.

Indiscutiblemente una gran limitante en el estudio lo constituyó el poco conocimiento que los gerentes tienen en torno al tema de la gestión del conocimiento. De igual manera, los exiguos recursos destinados a este tipo de investigaciones hace que el proceso no se pueda ahondar un poco más en otro tipo de compañías como las micro y pequeñas empresas de la región de Sugamuxi, pues su número en términos reales es muy alto. Por otro lado, la labor gerencial propia de los ejecutivos impidió que el estudio se realizara en un menor tiempo, dado que la resolución del instrumento guiado requería de un tiempo prudencial de los gerentes, los cuales por sus actividades cotidianas les era difícil completar en una sola sesión.

La propuesta del modelo de gestión, en cuanto tiene que ver con las medianas y grandes empresas del Valle de Sugamuxi, muestra la importancia de crear, difundir, usar y transferir el conocimiento existente en toda la organización en pro del mejoramiento del desarrollo organizacional, que para tal fin, queda a consideración de los empresarios y gerentes de las empresas del Valle de Sugamuxi, los respectivos re-ajustes, teniendo en cuenta las características de la organización de cada una de las empresas a su cargo.

Por último, la investigación permitió concluir que la mayoría de los gerentes del Valle de Sugamuxi aún emplean, en su gestión, disciplinas que carecen de participación de los colaboradores, utilización de tecnologías adecuadas, falta de inversión en II\&D y escasa generación en cuanto a la innovación y creación de productos y /o servicios, todo lo cual llama la atención sobre la importancia de la gestión del conocimiento en estas organizaciones como un medio para potencializar sus recursos y capacidades. 


\section{Referencias bibliográficas}

Arbonies, A. (2006). Conocimiento para Innovar La Sociedad del Conocimiento. Madrid: Díaz de Santos.

Bernal, M. (2005). Caracterización de la Gestión del Conocimiento y Propuestas para su Mejoramiento en entidades del Nivel Central de la Administración Distrital de Bogotá. Tesis no publicada de Grado de Magíster en Administración, Universidad Nacional de Colombia, Facultad de Ciencias Económicas, Bogotá, Colombia. Carrillo, F. (2006). De la organización del conocimiento a la sociedad del conocimiento. Revista semana internacional de la gestión del conocimiento. Memorias Semana Internacional de la Gestión del Conocimiento. Barranquilla, Colombia: ESAN.

Clavijo, M. (2011). Cómo retener el Talento. Gerente, 156, 44-47.

Clemmons, M. (2002). Knowledge Management, Exploring the Oxymoron (Vol. 1). Estados Unidos: Alpha Books.

Davenport, T. (1997). Some principles of knowledge management. Recuperado el 18 de diciembre de 2010 , de http://www.strategy-business.com/ article/8776?gko=f91a7

David, P. \& Foray, D. (2002). Fundamentos económicos de la sociedad del conocimiento. Revista comercio Exterior, 52(6), 472-490.

De los Santos, S. (1997). Modelo de medición y generación de ventajas competitivas sostenibles en el ámbito de la iniciativa, respuesta eficiente al consumidor (efficient consumer response). Madrid: Universidad Rey Juan Carlos.

Díaz, J. (2003). Modelo de Gestión (gestión del conocimiento) aplicado a la universidad pública en el Perú. Recuperado el
28 diciembre de 2010, de http://sisbib. unmsm.edu.pe/bibvirtual/tesis/Basic/ Diaz_MJ/Contenido.htm

Díaz, L. (2002). Estadística Multivariada, Análisis de factores comunes y únicos. Bogotá: UPTC.

Drucker, P. (1993). Post Capitals Society. Oxford, uk: Butterword Heineman.

Durango, C. (2005). Fundamentación Epistemológica de los estudios organizacionales. Medellín, Colombia: Universidad Pontificia Bolivariana.

Fernández, F. \& Cordero, A. (2010). Proceso de Gestión del conocimiento en Carabobo (Venezuela) y Tamaulipas (México). Revista Pensamiento y Gestión, 28, 132-154. Recuperado el 18 enero de 2011, de http://www.gestionconocimiento. com/?gclid=CPTg $3 \mathrm{~L}_{7}$ sipECFQT-lgodaAFXHw

Fresno, C. (2001). A favor de la gestión del conocimiento. Recuperado el 21 de mayo de 2011.

García, F. \& Cordero, A. (2008). Los equipos de trabajo: una práctica basada en la gestión del conocimiento. Visión gerencial, 7(1), 45-58. Recuperado el 21 de mayo de 2011, de http://www.saber.ula.ve/bitstream/123456789/25173/2/articulo4.pdf García, F., Domínguez, A.L. \& Sánchez, M. (2005). Fundamentos teórico-econômicos da gestão do conhecimento. Organizações em contexto, 1(2), 119-134. Recuperado el 21 de mayo de 2011.

García, I. (2002). La Gestión del Conocimiento el nuevo Paradigma de las Empresas. Recuperado el 28 de febrero de 2008 , de http://cdt.robotiker.es/cdt/articulos/ mostrar_pdf.jsp?id=1

Garrido, R. (2002). Diseño de un modelo de Gestión del Conocimiento para la Unellez, Universidad Nacional Experimental de los Llanos Occidentales Ezequiel ZamoraUNELLEZ. Recuperado el 2 de abril de 
2011, de http://www.monografias.com/ trabajos17/unellez/unellez.shtml

González, J. (2009). Aplicación y desarrollo de la gestión del conocimiento de los grupos de investigación de la UPTC. Bogotá: Universidad Nacional de Colombia.

Grau, A. (2001). Herramientas de Gestión del Conocimiento, Fundación Iberoamericana del Conocimiento. Recuperado el 2 de abril de 2011, de_http://docencia.udea.edu. co/ingenieria/semgestionconocimiento/ documentos/Mod7_HerrTec.pdf_

Inche, M. \& Chung, P. (2004). Indicadores de gestión del conocimiento en la Facultad de Ingeniería Industrial. Recuperado el 23 enero 2011, de http://redalyc.uaemex.mx/ src/inicio/ArtPdfRed.jsp?iCve $=81670207$

Ley 1111, Por la cual se modifica el estatuto tributario de los impuestos administrados por la Dirección de Impuestos y Aduanas Nacionales, Congreso de la Republica, 2006. Disponible en http://www.actualicese. com/normatividad/2006/12/27/ley-1111de-27122006/

Medellín, E. (2008). El conocimiento y su administración en las empresas. En J. Micheli, E. Medellín, J. Jasso \& A. Hidalgo (Coords.), Conocimiento e innovación: retos de la gestión empresarial. México: Plaza y Valdés.

Moreno, L. (2000). Región y Sociedad, Los negocios en la era digital (Vol.1). México: Plaza \& Janes.

Nonaka, I. \& Takeuchi, H. (1995). The knowledge creating company. New York, NY: Oxford University Press.

Pavez, A. (2001). Modelo de Implantación de Gestión del de la Información para la Generación de Ventajas Competitivas. Tesis de pregrado no publicada, Universidad Técnica Federico Santa María, Valparaíso, Chile.

Plaz, R. (2003). Gestión del Conocimiento: Una visión integradora del aprendizaje or- ganizacional. Recuperado el 5 de octubre de 2010, de http://www.madrimasd.org/ revista/revista18/tribuna/tribuna2.asp Polanyi, M. (1962). Personal Knowledge: an evolutionary approach. Oxford, UK: Clarendon press.

Rodríguez, A., Araujo, A. \& Urrutia, J. (2001). La Gestión del Conocimiento Científico-Técnico en la Universidad: un Caso y un Proyecto, Universidad del País Vasco-Euskal Erico Unibertsitatea (UPV/ EHU). Cuadernos de Gestión, 1(1), 13-3.

Shanhong, T. (2002). Gestión del Conocimiento en las Bibliotecas del siglo XXI, Características de la Gestión del Conocimiento en Bibliotecas. Recuperado el 5 de mayo de 2011, de http://www.ifla.org/Iv/ ifla66/papers/057-110s.htm

Serradell, E. \& Pérez, A.J. (2000). La Gestión del Conocimiento en la Nueva Economía, concepto de la Gestión del conocimiento. Recuperado el 5 de octubre de 2010, de http://www.uoc.edu/dt/20133/index. html\#bibliografia

Sveiby, K. (1997). The New Organizational wealth, Managing and Measuring Knowledge- based assets. Brisbane, Australia: Berrett Koehler.

Sveiby, K. (1998). Measuring Intangibles and Intellectual Capital - An Emerging First Standard. Recuperado el 18 enero de 2011, de http://www.sveiby.com/articles/ EmergingStandard.html

Swan, J. \& Scarbrough, H. (2001). Knowledge management: concepts and controversies. Journal of Management Studies, 38(7), 913-921.

Valhondon, D. (2003). Gestión del Conocimiento del Mito a la Realidad. Madrid: Díaz De Santos.

Vargas, A. \& Moreno, M. (2002). La gestión del conocimiento en las organizaciones. Investigaciones sobre la Aplicación del Conocimiento. Recuperado el 25 de febrero 
de 2010, de http://tmstudies.net/index. $\mathrm{php} / \mathrm{ectms} /$ article/download/12/128

Zorrilla, H. (1997). La gerencia de conocimiento y la gestión tecnológica. Programa de Gestión tecnológica. Recuperado el 18 de enero de 2011, de http://www.sht.com. ar/archivo/Management/conocimiento. htm 